\title{
A New Image Noise Detection Approach using Morphology and Partial Differential Equations
}

\author{
Yubin Li \\ Gannan Normal University, Ganzhou, China \\ liyubin2201@163.com
}

\begin{abstract}
In recent years, the theory of partial differential equations (PDE) for its rigorous mathematical theory foundation has been widely used in the fields of image processing. Medical imaging seeks to reveal internal structures hidden by the skin and bones, as well as to diagnose and treat disease. In order to detect the noise in the medical images, many models are studied, but the noises in medical images are much more complex than typical images. This paper introduces a new image noise detection approach using morphology and partial differential equations where are based on the morphology reconstruction with anisotropic diffusion to make full use of the advantage of Catte model. This proposed approach has been tested with the biomedical cell images with comparing with the Catte model, PM model and Canny model. The experimental results show that this proposed approach outperforms the other three models in terms of defined indicator and efficiency.
\end{abstract}

Keywords: Image Processing, Noise Detection, Morphology, Partial Differential Equation, Diffusion

\section{Introduction}

Bioinformatics is a branch of image processing bio-engineering disciplines, including biological information processing technology, bio-image processing and analysis, which are disciplines in the field of bio-engineering direction of the fastest growing [1]. Bioinformatics dedicated image analysis to extract information from the biometric image or a digital image of biological sequences in the field of life sciences which have a wide range of applications. However, there is often a certain image noise. For subsequent image analysis and processing, the input image typically must be de-noised to improve the image quality. Bioinformatics de-noising not only requires to remove blur and image noise, but also needs to keep the details of the image [2]. Traditional filtering methods are difficult to deal with such problems.

In recent years, the theory of partial differential equations (PDE) for its rigorous mathematical theory foundation has been widely used in the fields of image processing. A PDE is a differential equation that contains unknown multivariable functions and their partial derivatives, which are used to formulate problems involving functions of several variables, and are either solved by hand, or used to create a relevant computer model [3]. PDEs can be used to describe a wide variety of phenomena such as sound, heat, electrostatics, electrodynamics, fluid flow, elasticity, or quantum mechanics. These seemingly distinct physical phenomena can be formalized similarly in terms of PDEs [4]. Just as ordinary differential equations often model one-dimensional dynamical systems, partial differential equations often model multidimensional systems. PDEs find their generalization in stochastic partial differential equations.

Images taken with both digital cameras and conventional film cameras will pick up noise from a variety of sources. Many further uses of these images require that the noise will be (partially) removed - for aesthetic purposes as in artistic work or marketing, or for practical purposes such as computer vision. In real-world photographs, the highest spatial- 
frequency detail consists mostly of variations in brightness ("luminance detail") rather than variations in hue ("chroma detail") [5]. Since any noise reduction algorithm should attempt to remove noise without sacrificing real detail from the scene photographed, one risks a greater loss of detail from luminance noise reduction than chroma noise reduction simply because most scenes have little high frequency chroma detail to begin with. In addition, most people find chroma noise in images more objectionable than luminance noise; the colored blobs are considered "digital-looking" and unnatural, compared to the grainy appearance of luminance noise that some compare to film grain [6]. For these two reasons, most photographic noise reduction algorithms split the image detail into chroma and luminance components and apply more noise reduction to the former.

Medical imaging is the technique, process and art of creating visual representations of the interior of a body for clinical analysis and medical intervention. Medical imaging seeks to reveal internal structures hidden by the skin and bones, as well as to diagnose and treat disease. Medical imaging also establishes a database of normal anatomy and physiology to make it possible to identify abnormalities [7]. Although imaging of removed organs and tissues can be performed for medical reasons, such procedures are usually considered part of pathology instead of medical imaging. As a discipline and in its widest sense, it is part of biological imaging and incorporates radiology which uses the imaging technologies of X-ray radiography, magnetic resonance imaging, medical ultrasonography or ultrasound, endoscopy, elastography, tactile imaging, thermography, medical photography and nuclear medicine functional imaging techniques as positron emission tomography [8].

Measurement and recording techniques which are not primarily designed to produce images, such as electroencephalography (EEG), magnetoencephalography (MEG), electrocardiography (ECG), and others represent other technologies which produce data susceptible to representation as a parameter graph vs. time or maps which contain information about the measurement locations. In a limited comparison these technologies can be considered as forms of medical imaging in another discipline. Up until 2010, 5 billion medical imaging studies had been conducted worldwide. Radiation exposure from medical imaging in 2006 made up about $50 \%$ of total ionizing radiation exposure in the United States [9].

In the clinical context, "invisible light" medical imaging is generally equated to radiology or "clinical imaging" and the medical practitioner responsible for interpreting (and sometimes acquiring) the images is a radiologist [10]. "Visible light" medical imaging involves digital video or still pictures that can be seen without special equipment. Dermatology and wound care are two modalities that use visible light imagery. Diagnostic radiography designates the technical aspects of medical imaging and in particular the acquisition of medical images [11]. The radiographer or radiologic technologist is usually responsible for acquiring medical images of diagnostic quality, although some radiological interventions are performed by radiologists. While radiology is an evaluation of anatomy, nuclear medicine provides functional assessment.

In order to detect the noise in the medical images, many models are studied such as Canny algorithm, wavelet model, and so on. However, the noises in medical images are much more complex than typical images. This paper introduces a new image noise detection approach using morphology and partial differential equations where are based on the morphology reconstruction with anisotropic diffusion to make full use of the advantage of Catte model.

\section{Basic Catte Model}

Catte model is an anisotropic diffusion model which was proposed by Catte and Morel. In this model, the diffusion coefficient $g(|\nabla u|)$ is replaced by $g\left(\left|\nabla u_{\sigma}\right|\right)$, $u_{\sigma}=G_{\sigma} \times u$ in the $P M$ equation. Using the diffusion coefficient, the edge of image 
is estimated. Based on the estimation, the diffusion coefficient is calculated for determining the diffusion degree so as to reduce the estimation error of the noises. The Catte diffusion model is:

$$
\begin{aligned}
& \frac{\partial u}{\partial t}=\operatorname{div}\left(g\left(\left|\nabla G_{\sigma} \times u\right|\right) \nabla u\right) \\
& u(x, y, 0)=u_{0}(x, y)
\end{aligned}
$$

Where $G_{\sigma}$ is the Gaussian core function:

$$
G_{\sigma}=\frac{1}{4 \pi \sigma} e^{-\frac{x^{2}+y^{2}}{4 \sigma}}
$$

$\left|\nabla G_{\sigma} \times u\right|$ presents the mod of gradient of the image with the filter scalability of $\sigma . u(x, y, 0)$ is the original image. The discrete of (1) could be obtained as:

$$
u_{p}^{\prime}=u_{p}^{\prime}+\lambda \sum_{q \in \eta_{p}}\left(\alpha_{p}^{\prime}+\alpha_{q}^{\prime}\right) \nabla u_{p, q}^{\prime}
$$

Where, $\alpha_{q}^{\prime}$ is the approximation of $g\left(\left|\nabla G_{\sigma} \times u\right|\right)$. In the Catte model, gradient $\nabla u$ is got from the calculation of the diffusion coefficient of an image using Gaussian filter convolution. The diffusion coefficient $g\left(\left|\nabla G_{\sigma} \times u\right|\right)$ is able to avoid the extra diffusion at the boundary, solving the well-posedness of $P M$ equation. The success attributes to the choosing of a suitable $\sigma$ which is used for normalizing factors because it is difficult to determine the scale $\sigma$ of Gaussian core. If $\sigma$ is too small, the estimation is not precise. Thus, the diffusion process will be emanative. If $\sigma$ is too large, the smoothness will be over. Additionally, the computation time will be costful when the Catte model carries out the convolution of Gaussian core before the diffusion.

There are some missing of the edge details after processing by the Catte model. That causes the unreality of the image $[4,8]$. The reason is that the peak edge characteristics and narrow edge characteristics are ignored during the diffusion procedure [3]. Therefore, $|\nabla u|$ could be replaced by $\left|\nabla u_{\sigma}\right|^{2}+u_{x x}^{2}+u_{y y}^{2}$. The heterogeneous diffusion equation is:

$$
\frac{\partial u}{\partial t}=\operatorname{div}\left(g\left(\left|\nabla G_{\sigma} \times u\right|^{2}+\left(G_{\sigma} \times u_{x x}\right)^{2}+\left(G_{\sigma} \times u_{y y}\right)^{2}\right) \nabla u\right.
$$

Where $u(x, y, 0)=u_{0}(x, y)$ and $g=\operatorname{sqrt}(x)$.

Using the improved diffusion equation, the second derivative of image pixels are adopted for calculating the diffusion coefficient. However, the disadvantage of this model is that, for evenly distribution noise and non-strong Gaussian noise, it performs well. However, strong Gaussian noise, for example the noise from medical images, the processing ability is weak due to its reservation of some peak edge pixels [12]. Thus, this paper introduces a morphology and partial differential equation integrated model for addressing this problem.

\section{New Model for Noise Detection based on Morphology and Partial Differential Equations}

\subsection{Morphology Re-construction}

Morphology re-construction is an important part in the morphological image processing, which is based on two images. The processing is iterative procedure which will be continuous until the image pixels are not changeable. Morphology re- 
construction is based on the mask image feature to label marker image. The initial points are the peak value or high value in the image. Let $M$ is the mask image, $R$ is the marker image. Morphology re-construction could be defined as:

$$
\operatorname{rec}_{\oplus}(M, R)=\underset{n \in N}{V} R_{n}
$$

$R_{n}, n \in N$ is an increasing sequence, whose iteration is defined as $R_{0}=R \wedge M$, $\forall n \in N, R_{n+1}=\left(R_{n} \oplus V\right) \wedge m$. Where $V$ is the neighbor area of original image. $\wedge$ is the upper boundary factor. Based on the re-construction, we propose some definitions in this paper for facilitating the image processing:

Definition 1. The morphology re-construction based on gradual erosion is defined as:

$$
\operatorname{rec}_{\Theta}(M, R)=\underset{n \in N}{\wedge} R_{n}
$$

Where $R_{0}=R \vee M, \forall n \in N, R_{n+1}=\left(R_{n} \Theta V\right) \vee m, \vee$ is the down boundary factor.

Definition 2. The morphology re-construction based on open operator is defined as:

$$
\gamma_{\text {rec }}(X)=\operatorname{rec}_{\oplus}(X, \gamma(X))
$$

Where $\gamma$ is the morphology open operator.

Definition 3. The morphology re-construction based on close operator is defined as:

$$
\varphi_{\text {rec }}(X)=\operatorname{rec}_{\Theta}(X, \varphi(X))
$$

Where $\varphi$ is the morphology close operator.

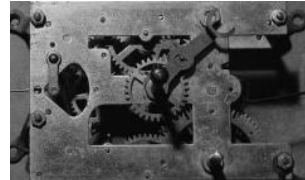

(a)Original Image

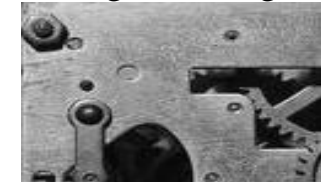

(e)Sub-region of (a) 3of e

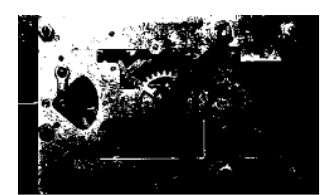

(b)Definition 1

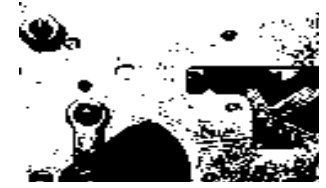

(f)Definition 1 of $\mathrm{e}$

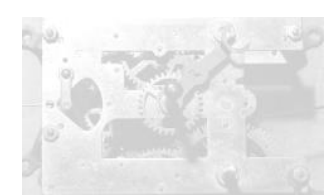

(c)Definition 2

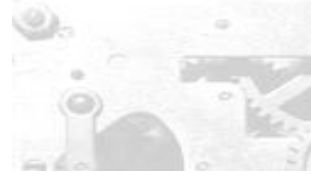

(g)Definition 2 of e

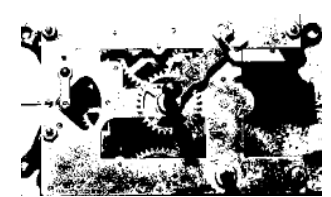

(d)Definition 3

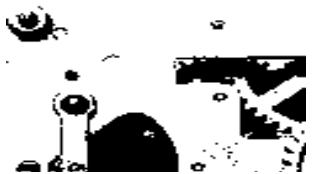

(h)Definition

Figure 1. Morphology Re-construction Images

According to the definitions, Figure 1 shows a result on different morphology reconstruction images based on the definitions mentioned above. The Figure 1 (a) shows an original image which is under processed by the above three definitions of morphology re-construction which are used for the noise detection. From the first experiment, Figure 1 (b)(c)(d) show the results by using the re-construction method using gradual erosion, open and close operator. It could be observed that these definitions will not cause the shape change of the original image. In order to examine the ability of morphology approaches on detecting the noise and remove them, we carried out the second experiment that enlarges the original image in (a). Figure 1 (e) is a sub-region enlarged from (a), (f)(g)(h) are from using the same morphology re-construction approach on (e). It could be found that the open and close operator can reduce some noises and keep the edge structure.

For medical image, the open and close morphology re-construction approaches are able to remove the noses hidden in the background. The small burr could be 
detected and smoothened as well in detail. Thus, the image gray degree could be kept to implement this approach in practice.

\subsection{New Partial Diffusion Equations}

Based on the above definitions, this section presents a new partial diffusion equation to remove the isolated pixel without changing the edge. The model is expressed as:

$$
\frac{\partial I u}{\partial t}=\operatorname{div}\left(g\left(|\nabla u|^{2}+\left(I u_{x x}\right)^{2}+\left(I u_{y y}\right)^{2}\right) \nabla I u\right)
$$

Where $u(x, y, 0)=u_{0}(x, y), g=g(\operatorname{sqrt}(x))$, and $I u$ is the re-constructed image based on the open and closed operator from original image $u$. The gradient $\nabla I u$ in this model is obtained from the morphology re-construction. In this model, the minor change is detected and removed.

Let $\varphi(c)=g(c) I u_{x}, c=\left(I u_{x}\right)^{2}+\left(I u_{x x}\right)^{2}$, assume that the edge is parallel with direction $y$. Then the equation (10) could be converted into:

$$
I u_{t}=\frac{\partial}{\partial x}(\varphi(c))=\varphi(c) I u_{x x}=2 g^{\prime}(c)\left(I u_{x}+I u_{x x x}\right) I u_{x} I u_{x x}+g(c) I u_{x x}
$$

Then, $\frac{\partial}{\partial t}\left(I u_{x}\right)=\frac{\partial}{\partial t}\left(I u_{x}\right)=\frac{\partial}{\partial t}\left(\frac{\partial}{\partial x} \varphi(c)\right)$.

$$
\begin{aligned}
\frac{\partial}{\partial t}\left(I u_{x}\right)= & 4 g^{\prime \prime}(c)\left(I u_{x}+I u_{x x x}\right)^{2} I u_{x}\left(I u_{x x}\right)^{2} \\
& +2 g^{\prime}(c)\left(I u_{x x}+I u_{x x x}\right) I u_{x} I u_{x x} \\
& +4 g^{\prime}(c)\left(I u_{x}+I u_{x x x}\right)\left(I u_{x x}\right)^{2} \\
& +2 g^{\prime}(c)\left(I u_{x}+I u_{x x x}\right) I u_{x} I u_{x x x} \\
& +g(c) I u_{x x x}
\end{aligned}
$$

When $I u_{x x}=0$,

$$
\frac{\partial}{\partial t}\left(I u_{x}\right)=2 g^{\prime}\left(I u_{x}^{2}\right)\left(I u_{x}+I u_{x x x}\right) I u_{x} I u_{x x x}+g\left(I u_{x}^{2}\right) I u_{x x x}
$$

Assume that $g(x)=\frac{1}{1+\frac{x}{k^{2}}}$, then (13) could be converted to:

$$
\frac{\partial}{\partial t}\left(I u_{x}\right)=-\frac{\left(\frac{I u_{x}}{k}\right)^{2}}{\left(1+\frac{I u_{x}}{k^{2}}\right)^{2}} I u_{x x x}-\frac{\left(\frac{I u_{x x x}}{k}\right)^{2}}{\left(1+\frac{I u_{x}}{k^{2}}\right)^{2}} I u_{x}+\frac{1}{\left(1+\frac{I u_{x}}{k^{2}}\right)} I u_{x x x}
$$

In the flat area, $\left|I u_{x x x} \square\right| I u_{x} \mid$, the gradient is mainly controlled by $I u_{x}$. When $I u_{x}>k, \frac{\partial}{\partial t}\left(I u_{x}\right)>0, I u_{x}$ increases slowly. Oppositely, if $I u_{x}<k, \frac{\partial}{\partial t}\left(I u_{x}\right)<0$, $I u_{x}$ decreases quickly. Using this manner, it is able to filter the noises hidden in medical image and keep the edge details. For images, the peak and narrow edge area will not expend. That means it will not enhance the noise, resulting more clearly processed images. 


\subsection{Parameter Setting}

The image noise detection aims to highlight the edge boundary. In order to increase the diffusion speed, this paper uses different diffusion functions with different gradient. For small gradient, the bigger diffusion speed will be used to connect the area with neighbor gray value [13]. For the bigger gradient area, small diffusion speed will be used to enhance the image details on boundary. That could be expressed as:

When $\left|\nabla I u_{x}\right|<k, \quad g_{1}(|\nabla I u|, t)=\frac{1}{1+\left(\frac{|\nabla I u|}{k}\right)^{2}}$, then when $\left|\nabla I u_{x}\right|>k$, $g_{2}(|\nabla I u|, t)=\frac{1}{1+\frac{|\nabla I u|}{k}}$. The diffusion coefficient $g_{1}(\square)$ can keep the smoothness of the edge smoothness, while the diffusion coefficient $g_{2}(\square)$ can avoid the unnecessary edge comparison enhancement. When $\left|\nabla I u_{x}\right|=k, g_{1}(\square)$ and $g_{2}(\square)$ have the same value. In order to improve the whole heterogeneous diffusion with more smooth, in the area of $|\nabla I u|=k \pm \Delta k$, the diffusion coefficient could be:

$$
g_{1}(|\nabla I u|, t)=\frac{1}{2\left(1+\left(\frac{|\nabla I u|}{k}\right)\right.}+\frac{1}{2\left(\left(1+\left(\frac{|\nabla I u|}{k}\right)^{2}\right)\right.}
$$

In the other side, from the essence of diffusion, with the iteration of diffusion increasing, the gradient of image will become small. Because the condition of keeping edge of an image is $|\nabla I u|>k$, the value of $k$ should be decreased. The threshold of parameter could be defined as a function of time $t$,

$$
k_{t+1}=\frac{1}{\gamma} k_{t}, \gamma \in[1,+\infty)
$$

From (16), with the increasing of $t$, the threshold will become smaller and smaller. Thus, this can reduce the unnecessary diffusion, greatly cutting down the cost of time on noise detection in image processing. When $k \rightarrow 0$, the diffusion will reach the stable status.

\section{Experimental Results and Discussions}

The experiment is carried out firstly through numerical solution, the proposed heterogeneous diffusion function could be discrete as:

$$
u_{i, j}^{t+1}=u_{i, j}^{t}+\lambda\left[C_{N}^{t} \square D_{N} u_{i, j}^{t}+C_{S}^{t} \square D_{S} u_{i, j}^{t}+C_{E}^{t} \square D_{E} u_{i, j}^{t}+C_{W}^{t} \square D_{w} u_{i, j}^{t}\right]
$$

Where $\lambda=\Delta t, 0 \leq \lambda \leq \frac{1}{4}, D_{N} u_{i, j}^{t}, D_{S} u_{i, j}^{t}, D_{E} u_{i, j}^{t}$, and $D_{W} u_{i, j}^{t}$ are the four neighbor difference:

$$
D_{N} u_{i, j}^{t}=u_{i-1, j}^{t}-u_{i, j}^{t}, D_{S} u_{i, j}^{t}=u_{i+1, j}^{t}-u_{i, j}^{t}, D_{E} u_{i, j}^{t}=u_{i, j+1}^{t}-u_{i, j}^{t}, D_{W} u_{i, j}^{t}=u_{i, j-1}^{t}-u_{i, j}^{t}
$$

In order to avoid the value of $|\nabla I u|^{2}+\left(I u_{x x}\right)^{2}+\left(I u_{y y}\right)^{2}$ becoming small, partial derivative solution with first step cannot use the central difference. For space second step partial derivative, the forward difference is obtained from first step partial derivative of two half-point, thus, we can get: 


$$
\begin{aligned}
& I u_{x x}^{t}=I u_{i, j-1}^{t}-2 I u_{i, j}^{t}+I u_{i, j+1}^{t} \\
& I u_{y y}^{t}=I u_{i-1, j}^{t}-2 I u_{i, j}^{t}+I u_{i+1, j}^{t}
\end{aligned}
$$

The conductivity factors of four neighbor area are $C_{N}^{t}, C_{S}^{t}, C_{E}^{t}$, and $C_{W}^{t}$.

Let $\alpha_{i, j}^{t}=\left(I u_{x x}^{t}\right)_{i, j}^{2}+\left(I u_{y y}^{t}\right)_{i, j}^{2}$

$$
=\left(I u_{i, j-1}^{t}-2 I u_{i, j}^{t}+I u_{i, j+1}^{t}\right)^{2}+\left(I u_{i-1, j}^{t}-2 I u_{i, j}^{t}+I u_{i+1, j}^{t}\right)^{2}
$$

Then,

$$
\begin{aligned}
& C_{N}^{t}=g\left(\left|\left(\nabla I u^{t}\right)_{i-1, j}\right|^{2}+\alpha_{i, j}^{t}\right) \\
& C_{S}^{t}=g\left(\left|\left(\nabla I u^{t}\right)_{i+1, j}\right|^{2}+\alpha_{i, j}^{t}\right) \\
& C_{E}^{t}=g\left(\left|\left(\nabla I u^{t}\right)_{i, j+1}\right|^{2}+\alpha_{i, j}^{t}\right) \\
& C_{W}^{t}=g\left(\left|\left(\nabla I u^{t}\right)_{i, j-1}\right|^{2}+\alpha_{i, j}^{t}\right)
\end{aligned}
$$

Secondly, in order to evaluate the feasibility and practicality of the proposed model in this paper, the experiment uses the biological cell image and MRI image for carrying out the testing. The time $\Delta t$ and $\gamma$ could be set as $0 \leq \Delta t \leq \frac{1}{4}$ and $\gamma=2$. The threshold $k_{0}$ is obtained from the high threshold from the Canny factor.
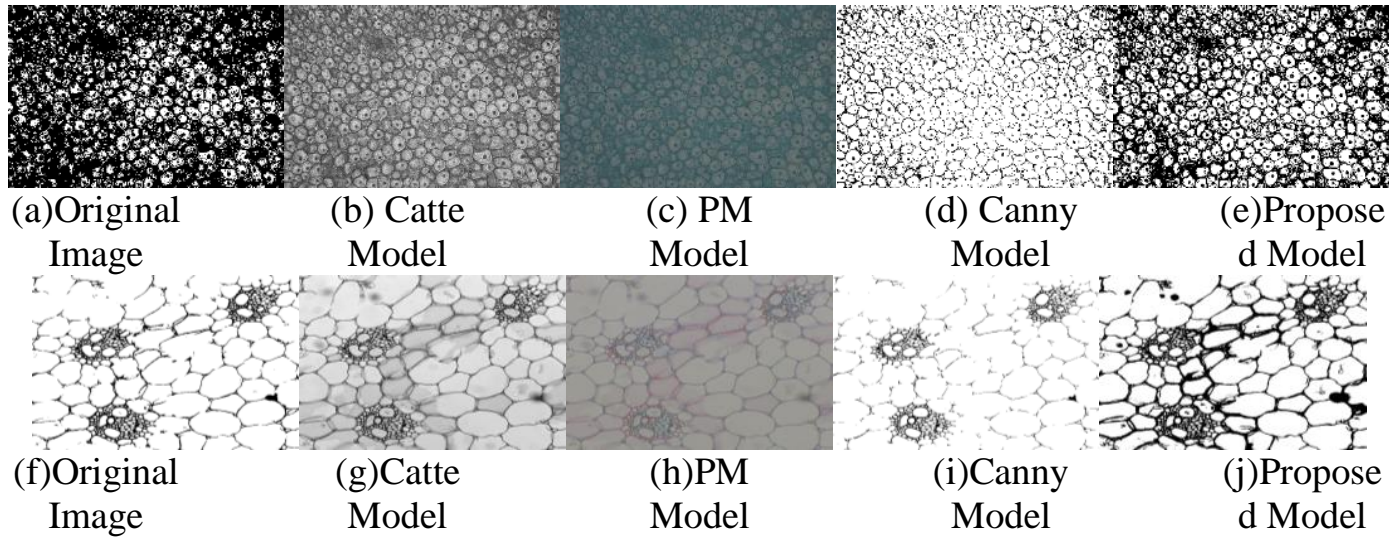

Figure 2. Experiment Results

Figure 2 shows the experiment results from using the cell images. Two types of cell images are used for demonstrating the noise detection. Figure 1 (a) is an original image with $20 \%$ Gaussian noise. (b)(c)(d) are the results processed by using Catte model, PM model, and Canny model. It could be observed that, Catte and PM model has the ability for getting a smooth image after removing the noise. However, the diffusion of the edge and total image is over-expended that the images are still ambiguous for getting the characteristics of cells. (d) shows the Canny model on removing the noises and highlight the edge of cells. It could be observed that the effectiveness of this model is much better than Catte and PM model. However, the processed image is too bright because the cell is different from other images. The cell image will have a liquid part with a core in the center and the cell edge is so tiny. (e) shows the result from using the morphology and partial differential equation based model. It could be found that this approach is able to detect and remove the noise efficiently and effectively as well as to highlight the edge characteristics. Using the images, the deteriorated cells could be observed and used for diagnosis. 
The second type of experiment is based on a biomedical neural cell image which contains three main neural cells. Figure 2 (f) contains the original image with $10 \%$ white noise. The proposed model is compared with Catte model, PM model and Canny model. It could be observed from the results, ( $\mathrm{j}$ ) image is well processed by removing the white noise from the image and the neural cell edges are highlighted. The characteristics of them are emphasized with obvious efficiency.

The final experiment is carried out for examining the noise detection of heterogeneous diffusion equations. The Peak Signalto Noise Ratio (PSNR) and normalized root mean square error (NMSE) are used for quantitatively evaluating the models.

$$
\begin{aligned}
& P S N R=10 \log _{10}\left(\frac{\left(2^{n}-1\right)^{2}}{\frac{1}{M \times N} \sum_{i} \sum_{j}\left[I u_{o}(i, j)-I u_{r}(i, j)\right]^{2}}\right) \\
& N M S E=\frac{\sum_{i=1}^{M} \sum_{j=1}^{N}\left[I u_{o}(i, j)-I u_{r}(i, j)\right]^{2}}{\sum_{i=1}^{M} \sum_{j=1}^{N}\left[I u_{o}(i, j)\right]^{2}}
\end{aligned}
$$

Where, $I u_{o}$ is the original image and $I u_{r}$ is the resulted image. $i, j$ present the image location. $M \times N$ is the image resolution, $n$ is the image color depth. Using these two indicators, the noise detection could be examined and PSNR is bigger and NMSE is smaller is better respectively. That indicates the capability of noise detection ability of different models.

Table 1. PSNR and NMSE of Different Models

\begin{tabular}{cccccc}
\hline $\begin{array}{c}\text { Gaussian } \\
\text { Deviation }\end{array}$ & Indicators & $\begin{array}{c}\text { Catte } \\
\text { Model }\end{array}$ & PM Model & $\begin{array}{c}\text { Canny } \\
\text { Model }\end{array}$ & $\begin{array}{c}\text { Proposed } \\
\text { Model }\end{array}$ \\
\hline \multirow{2}{*}{0.01} & PSNR & 23.432 & 24.552 & 26.878 & 31.673 \\
& NMSE $\left(10^{-4}\right)$ & 0.985 & 0.467 & 0.288 & 0.229 \\
0.02 & PSNR & 21.347 & 23.446 & 24.890 & 27.332 \\
& NMSE $\left(10^{-4}\right)$ & 1.987 & 1.335 & 1.021 & 0.383 \\
0.05 & PSNR & 18.899 & 20.329 & 23.658 & 25.662 \\
& NMSE $\left(10^{-4}\right)$ & 6.430 & 5.788 & 4.442 & 3.881 \\
\hline
\end{tabular}

From Table 1, the evaluation indicators of PSNR and NMSE are presented. Gaussian Deviation is set with $0.01,0.02$, and 0.05 respectively for examining Catte model, PM model, Canny model and the approach proposed in this paper. The above table could be expressed as several aspects. Firstly, comparing the PSNR under different Gaussian deviations, it could be observed that, the proposed model in this paper has the maximum values: $31.673,27.332$, and 25.662 respectively. That means the proposed method has better noise detection ability than the other three ones. Secondly, comparing the NMSE, the proposed model has the minimum values, which present the suitable diffusion degree for processing the image. Thirdly, when the Gaussian deviation increase, it is observed that the PSNR values for each model are decreasing; while the NMSE values for each model are increasing. However, the gradient for the increase of NMSE value is different. The Catte model has the biggest gradient, while the proposed model has the smallest gradient. That could be found the Catte model could detect the noise with quickest speed, which may cause the over-smoothness problem. 


\section{Conclusion}

This paper introduces a new image noise detection approach by integrating morphology and partial differential equation to process the biomedical images, which have some featured difficulties comparing with other typical images. In order to address the diffusion of edges in the image, morphology approach is used for controlling the diffusion speed and partial differential equation is used to control the efficiency and effectiveness of detection of the noises in the image. This proposed approach has been tested with the biomedical cell images with comparing with the Catte model, PM model and Canny model. The experimental results show that this proposed approach outperforms the other three models in terms of defined indicator and efficiency.

Future research directions should took into account so as to improve the implementation of this approach in practical applications. Firstly, the parameters in this model is based on the Canny threshold. It has not been compared with other manner. The question on how to find an optimal threshold $k$ could be examined by different impact factors using different operators. Secondly, the experiments carried out in this paper are based on two groups. More biomedical images will be used for testing the proposed model by comparing with other typical noise detection models or algorithms. So that, the optimal approaches could be figured out to process different images under different considerations [14]. To this end, suitable indicators should be defined first [15]. Finally, the proposed model could be integrated to an image processing system, which is able to assisting the end-users to make decisions on diagnosing the biomedical images in the future.

\section{References}

[1] T. Oinn, M. Addis, J. Ferris, D. Marvin, M. Senger, M. Greenwood, T. Carver, K. Glover, M. R. Pocock, A. Wipat, P. Li and Taverna, "A tool for the composition and enactment of bioinformatics workflows", Bioinformatics, vol. 20, (2004), pp. 3045-3054.

[2] B. Smolka and A. Chydzinski, "Fast detection and impulsive noise removal in color images", Real-Time Imaging, vol. 11, (2005), pp. 389-402.

[3] A. M. Wazwaz, "Partial Differential Equations: CRC Press", (2012).

[4] J. L. Eftang, A. T. Patera, and E. M. Rønquist, "An" hp" certified reduced basis method for parametrized elliptic partial differential equations", SIAM Journal on Scientific Computing, vol. 32, (2010), pp. 31703200.

[5] L. Kéïta, J. Guy, C. Berthiaume, L. Mottron, and A. Bertone, "An early origin for detailed perception in Autism Spectrum Disorder: biased sensitivity for high-spatial frequency information", Scientific reports, vol. 4, (2014).

[6] G. Jeon and E. Dubois, "Demosaicking of Noisy Bayer-Sampled Color Images with Least-Squares Luma-Chroma Demultiplexing and Noise Level Estimation", IEEE Transactions on Image Processing, vol. 22, (2013), pp. 146-156.

[7] S. Klein, M. Staring, K. Murphy, M. A. Viergever, and J. P. Pluim, "Elastix: a toolbox for intensitybased medical image registration", IEEE Transactions on Medical Imaging, vol. 29, (2010), pp. 196-205.

[8] A. Sotiras, C. Davatzikos, and N. Paragios, "Deformable medical image registration: A survey, Medical Imaging”, IEEE Transactions on, vol. 32, (2013), pp. 1153-1190.

[9] S. Bhavani and D. K. Thanushkodi, "A survey on coding algorithms in medical image compression", International Journal on Computer Science and Engineering, vol. 2, (2010), pp. 1429-1434.

[10] S. Gioux, H. S. Choi, and J. V. Frangioni, "Image-guided surgery using invisible near-infrared light: fundamentals of clinical translation", Molecular imaging, vol. 9, (2010), p. 237.

[11] R. Y. Zhong, Q. Y. Dai, T. Qu, G. J. Hu, and G. Q. Huang, "RFID-enabled Real-time Manufacturing Execution System for Mass-customization Production", Robotics and Computer-Integrated Manufacturing, vol. 29, (2013), pp. 283-292.

[12] F. Luisier, T. Blu, and M. Unser, "Image denoising in mixed Poisson-Gaussian noise, Image Processing", IEEE Transactions on, vol. 20, (2011), pp. 696-708.

[13] R. Y. Zhong, Q. Y. Dai, K. Zhou, and X. B. Dai, "Design and Implementation of DMES Based on RFID", $2^{\text {nd }}$ International Conference on Anti-counterfeiting, Security and Identification, (2008) 20-23 Aug Guiyang, China.

[14] R. Y. Zhong, G. Q. Huang, Q. Y. Dai, and T. Zhang, "Mining SOTs and Dispatching Rules from RFIDenabled Real-time Shopfloor Production Data", Journal of Intelligent Manufacturing, vol. 25, (2014), pp. 825-843. 
[15] Q. Y. Dai, R. Y. Zhong, G. Q. Huang, T. Qu, T. Zhang, and T. Y. Luo, "Radio frequency identificationenabled real-time manufacturing execution system: a case study in an automotive part manufacturer", International Journal of Computer Integrated Manufacturing, vol. 25, (2012), pp. 51-65.

\section{Author}

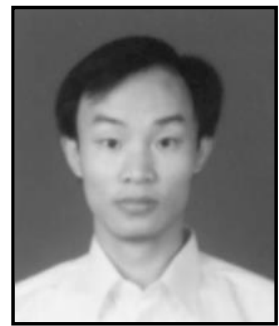

Yubin Li, he is a lecturer in Gannan Normal University. He graduated from Mathematics and Computer Science \& Technology, Gannan Normal University with bachelor degree in 2004 and master degree in software engineering from Yunnan University in 2009 respectively. His research direction includes image processing, computer applications, information management. He has published several papers in international/national journals and conference. 